\title{
Al-QAïda AU Maghreb islamiQue: LE TOURNANT DES RÉVOLUTIONS ARABES
}

Mathieu GUIDERE *

\section{INTRODUCTION}

Le vent révolutionnaire qui souffle sur le monde arabe depuis le début de l'année 2011 a bouleversé la donne géopolitique et affecté profondément tous les acteurs, y compris les groupes terroristes, à commencer par Al-Qaïda au Maghreb islamique (AQMI). Jamais il n'y a eu autant de messages et de communiqués officiels de l'organisation. En à peine six mois (janvierjuin 2011), le chef d'AQMI a émis quasiment un message par mois pour prendre position sur les événements en Tunisie, en Algérie, en Libye ou encore au Maroc; sans compter les messages diffusés par différents chefs de l'organisation après la mort de Ben Laden le 2 mai 2011. Bref, entre la colère de la rue arabe et la disparition du fondateur d'Al-Qaïda, la branche maghrébine se trouve aujourd'hui dans une situation délicate, obligée de se positionner par rapport à ces bouleversements et de revoir sa stratégie à tous les niveaux.

Après presque cinq ans d'existence (2006-2011), il est utile aujourd'hui de faire le point sur AQMI pour pouvoir anticiper ses évolutions futures. Pour cela, il est important d'aborder la question par une méthode raisonnée qui permette de se faire une idée précise et objective de la situation actuelle de l'organisation. Aussi, les analyses qui suivent sont issues d'une méthodologie de veille stratégique fondée sur un recoupement systématique de sources formelles et informelles en trois langues: arabe, français et anglais ${ }^{1}$.

\footnotetext{
* Mathieu Guidere est professeur d'islamologie et pensée arabe à l'université de Toulouse 2.

1. Voir à ce sujet la section intitulée « Éléments de méthode » dans M. Guidère, Le Choc des révolutions arabes, Paris, éditions Autrement, 2011, pp. 184-205. Voir aussi M. Guidère (dir.), Traduction et Veille stratégique multilingue, Paris, éditions Le Manuscrit, 2008, pp. 11-50.
} 
Cette méthodologie vise à comprendre le phénomène de l'intérieur en lui appliquant ses propres catégories et ses propres référents idéologiques, ce qui revient à interroger l'organisation à partir de ses propres postulats théoriques et pratiques, en évitant le point de vue ethnocentré. Le phénomène est ainsi décrit à l'aide de concepts et de termes propres à la culture de l'organisation, l'objectif étant de faire un état des lieux "vu de l'intérieur » comme aide à la décision politique ou militaire ${ }^{2}$.

\section{AQMI VUE DE L'INTÉRIEUR: LES CHIFFRES CLÉS (2007-2011)}

Il est possible de se faire une idée relativement précise d'AQMI «vue de l'intérieur » grâce à la grande quantité de documents diffusés par l'organisation elle-même, sous plusieurs formes et sur tous supports (publications rédigées en arabe, enregistrements audio, vidéos, sites web, blogs, forums, réseaux sociaux). À cette somme considérable de données, il faut appliquer les méthodes de vérification et de validation propres à la veille stratégique (analyse, recoupement, croisement des données issues de plusieurs langues et supports).

Les données qui suivent proviennent d'une stricte application de cette méthodologie pendant quatre ans (2007-2011). Rappelons à cet égard que l'acte d'allégeance à Ben Laden et à Zawahiri prêté par le chef de l'organisation, l'Algérien Abdelmalek Droukdal, alias Abou Moussab Abdelwadoud, est daté du 11 septembre 2006. Mais la création officielle d'AQMI, consécutive au choix du nom, date quant à elle du 11 janvier 2007, le communiqué officiel en arabe ayant été publié quelques jours plus tard sur les principaux forums djihadistes de l'époque.

\section{Effectifs d'AQMI (janvier 2007-juin 2011)}

\begin{tabular}{|c|c|c|c|}
\hline Année & $\begin{array}{c}\text { Total } \\
\text { des arrestations }\end{array}$ & $\begin{array}{c}\text { Total } \\
\text { des membres tués }\end{array}$ & $\begin{array}{c}\text { Total (tués, } \\
\text { arrêtés, repentis) }\end{array}$ \\
\hline 2007 & 280 & 299 & 605 \\
\hline 2008 & 291 & 210 & 550 \\
\hline 2009 & 376 & 260 & 679 \\
\hline 2010 & 298 & 242 & 564 \\
\hline 2011 (juin) & 52 & 32 & 117 \\
\hline
\end{tabular}

L'évolution des effectifs d'AQMI est déduite à partir du nombre total des membres de l'organisation qui ont été tués, blessés, arrêtés ou qui se sont rendus aux autorités. Les chiffres ci-dessous recoupent diverses sources tant officielles qu'officieuses. Ils montrent globalement que les effectifs d'AQMI

2. Cette méthodologie a été initialement développée au CREC Saint-Cyr, entre 2004 et 2007, dans le cadre du programme de recherches Radicalization Watch Project (RWP) et a donné lieu à la publication de la première étude de fond sur AQMI. Voir M. Guidère, Al-Qaïda à la conquête du Maghreb, Paris, éditions du Rocher, 2007. 
ont peu varié au cours des quatre dernières années, avec une estimation stable de plus de 1000 combattants, mais avec des pertes considérables de l'ordre de $50 \%$ des effectifs par an. En voici le détail:

Les pertes d'AQMI supposent un renouvellement équivalent de l'ordre de $50 \%$ des effectifs par an. Les données concernant les « nouvelles recrues » se déduisent à partir des documents internes de l'organisation (vidéos et photos), mais essentiellement pour les «Brigades du Sahara » (région sud). Fin 2010, les pays du Sahel fournissaient environ la moitié des membres de l'organisation, majoritairement composée de Mauritaniens (environ 150) et de Maliens (une centaine environ) - le reste des combattants étant composé de Nigériens, de Tchadiens et de Burkinabais, sans oublier quelques transfuges somaliens, soudanais et nigérians. Pour la région nord, les effectifs sont toujours davantage composés d'Algériens, mais il est quasiment impossible de déduire le nombre exact des membres issus du Maroc, de Tunisie et de Libye.

Il est certain, en revanche, que l'obtention du label d'Al-Qaïda en 2007 signe le début de l'internationalisation de l'organisation, tant du point de vue des combattants que des territoires ${ }^{3}$. Cette internationalisation est observable dans le détail des actions menées entre 2007 et 2011, la principale évolution opérationnelle étant l'adoption à grande échelle du kidnapping de ressortissants occidentaux.

\section{Actions d'AQMI (janvier 2007-juin 2011)}

\begin{tabular}{|c|c|c|c|c|}
\hline Actions & $\begin{array}{c}\text { Explosifs } \\
\text { improvisés } \\
\text { (IED) }\end{array}$ & $\begin{array}{c}\text { Embuscades, } \\
\text { accrochages }\end{array}$ & $\begin{array}{c}\text { Attentats } \\
\text { suicides }\end{array}$ & $\begin{array}{c}\text { Enlèvements } \\
\text { d'Occidentaux }\end{array}$ \\
\hline 2007 & 76 & 27 & 18 & 11 \\
\hline 2008 & 63 & 24 & 12 & 32 \\
\hline 2009 & 58 & 28 & 8 & 14 \\
\hline 2010 & 27 & 19 & 4 & 18 \\
\hline 2011 (juin) & 19 & 10 & 0 & 5 \\
\hline
\end{tabular}

Il apparaît clairement que l'année 2008 a été une année « faste » du point de vue du kidnapping d'étrangers. Dès lors, les "Brigades du Sahara » se sont mises à enlever des ressortissants des pays occidentaux, y compris le Canada et les États-Unis. Tous ont été libérés contre des rançons, à l'exception du Britannique Edwin Dyer (exécuté le 31 mai 2009) et du Français Michel Germaneau (exécuté le 25 juillet 2010) ${ }^{4}$.

3. Voir à ce sujet M. Guidère, "La tentation internationale d'Al-Qaïda au Maghreb ", Focus stratégique, n 12, 2009, http://www.ifri.org/?page=detailcontribution\&id=6162 (juin 2011).

4. Concernant ces deux exécutions, la version d'Al-Qaïda diffère des versions officielles données par les gouvernements britannique et français. 
L'augmentation des enlèvements et la focalisation, à partir d'août 2010, sur le kidnapping de ressortissants français ont mis en exergue la problématique du financement des activités de l'organisation. En effet, à partir de 2007 avec l'internationalisation d'AQMI s'est posée la question cruciale du financement de leurs activités. Selon les débats internes, cette question aurait été discutée et tranchée lors du Conseil des chefs (Majlis al-A'yân) de septembre 2008, au cours duquel deux positions auraient été défendues: d'un côté, les défenseurs des sources de financement " habituelles » dans le Sud (contrebande de divers produits sous la houlette de Moktar Belmoktar), convaincus qu'il faut se contentenr de ces activités pour ne pas s'attirer les foudres des Occidentaux; de l'autre, les tenants des enlèvements d'étrangers, menés par Abdelhamid Abou Zeid, qui non seulement estiment que les positions des premiers sont "lâches et couardes ", mais qui considèrent aussi les sources de financement habituelles comme "non conformes à l'Islam ". Les partisans de Belmoktar contestent quant à eux que les enlèvements d'Occidentaux puissent être qualifiés légitimement de " djihad »-argument avancé par Abou Zeid et ses lieutenants pour justifier le changement de stratégie de l'organisation. Ces derniers l'emportèrent finalement au prétexte que la nature de l'ennemi (« croisés »/ « infidèles ») justifie l'action ( djihad » légitime) ${ }^{5}$.

À la demande d'Abou Zeid, le Comité juridique d'AQMI planche à partir de 2008 sur une redéfinition des sources de financement du djihad « du point de vue islamique ». Ainsi par exemple, une distinction est faite entre deux grands modes de financement considérés comme "plus islamiques » que la contrebande: d'une part, la Jizya qui désigne une sorte de taxe de passage perçue principalement sur les marchandises qui transitent par les territoires contrôlés par les groupes affiliés à AQMI (y compris le trafic de drogue et d'êtres humains); et d'autre part, la Diyya qui désigne littéralement le «prix du sang » et qui correspond à la rançon perçue pour tout otage occidental enlevé par l'organisation.

En détail, cette "redéfinition islamique » des sources de financement permet de constater que l'enlèvement de ressortissants occidentaux apparaît désormais comme l'activité la plus « recommandable » et la plus « rentable » pour AQMI ${ }^{6}$.

\section{Financement d'AQMI (janvier 2007-juin 2011)}

Dans la théologie interne d'AQMI, les otages (Rahâ'in) sont ainsi assimilés à des "prisonniers de guerre » (Asrâ) et leur libération est soumise à des

5. À noter que cette position d'Abou Zeid, c'est-à-dire l'assimilation du kidnapping à une forme de djihad, a été légitimée par Ben Laden en personne, le 27 octobre 2010, lors de son "Message au peuple français », en reprenant à son compte l'enlèvement des cinq Français à Arlit au Niger.

6. Les chiffres figurant dans le tableau correspondent à ce qui est annoncé dans les demandes officieuses de l'organisation par le biais des intermédiaires locaux et autres négociateurs régionaux. 


\begin{tabular}{|c|c|c|c|c|}
\hline Sources & $\begin{array}{c}\text { Diyya (rançons } \\
\text { d'otages) }\end{array}$ & $\begin{array}{c}\text { Jizya (taxes de } \\
\text { transit) }\end{array}$ & $\begin{array}{c}\text { Tijara (trafic, } \\
\text { contrebande) }\end{array}$ & $\begin{array}{c}\text { Zakat (charité, } \\
\text { dons) }\end{array}$ \\
\hline 2007 & 11 millions & $10 \%$ & $\mathrm{NC}$ & $10 \%$ \\
\hline 2008 & 32 millions & $10 \%$ & $\mathrm{NC}$ & $10 \%$ \\
\hline 2009 & 14 millions & $10 \%$ & $\mathrm{NC}$ & $10 \%$ \\
\hline 2010 & 36 millions & $10 \%$ & $\mathrm{NC}$ & $10 \%$ \\
\hline 2011 -juin & 90 millions & $10 \%$ & $\mathrm{NC}$ & $10 \%$ \\
\hline
\end{tabular}

conditions qui relèvent, selon l'organisation, du « droit musulman de la guerre ». Tout est négociable dans le cadre de cette théologie: l'échange des "prisonniers », la fixation du "prix du sang », la libération contre " conversion », etc. Mais tout est également soumis aux règles d'allégeance: la logique interne à l'organisation voudrait que les enlèvements réalisés par celui qui prête allégeance (le vassal) soient récupérables et revendicables par celui qui reçoit le serment d'allégeance (le suzerain). Le «vassal » se met ainsi au service d'un chef plus puissant et agit pour son compte mais avec une grande liberté d'action et de commandement, avec parfois des revendications rétroactives des actions menées.

\section{LE FONCTIONNEMENT INTERNE D'AQMI}

L'intelligence culturelle (Cultural Intelligence) appliquée au phénomène terroriste permet de comprendre, de l'intérieur, comment fonctionne aujourd'hui AQMI. En effet, sur le terrain, il n'existe pas véritablement de hiérarchie établie définitivement ni de structure de commandement unique, mais un leadership éclaté et évolutif. Ce leadership est fondé sur le serment d'allégeance (Bay'a, Walâ') prêté entre individus influents sur un territoire particulier et à un moment donné: Droukdal dans le Nord, Yahya Abu Ammar dans le Sud, Abou Zeid dans le Sud-Est, Belmokhtar dans le Sud-Ouest, Abdelkrim le Touareg dans la région de Kidal au Mali.

Chacun de ces chefs est lié par un serment d'allégeance à un autre chef au-dessus de lui et à un certain nombre de petits caïds en-dessous de lui. Mais il ne connaît pas personnellement tous les combattants ni tous les membres des groupuscules dont les chefs (vassaux) ont prêté allégeance à un chef commun (suzerain). Ce système inclut les chefs de tribus et de clans sur l'ensemble de la région sahélienne.

En schématisant, on peut dire que chaque chef de groupe ne connaît en fait qu'un seul individu mais il engage, par son allégeance personnelle, tous les membres de son groupe, parce que ceux-ci lui ont fait allégeance à leur tour et que leur serment les lie, indirectement mais totalement, au chef d'AQMI, Droukdal en l'occurrence, et au-delà, au chef d'Al-Qaïda en personne (Ben Laden hier, Zawahiri aujourd'hui). Celui-ci pourrait, s'il le souhaitait, prendre quel que soit le moment le commandement de l'ensemble de ces combattants à distance, sans jamais les avoir connus ni rencontrés. Le chef local s'efface alors automatiquement au profit de son suzerain, en vertu des règles régissant le serment d'allégeance. 
Mieux, les actions de chaque combattant ayant prêté allégeance à un petit chef local peuvent être réalisées au nom de Droukdal ou de Zawahiri et revendiquées ensuite par eux parce que tous les membres de l'organisation visent le même objectif et adhérent aux mêmes règles.

Ces règles d'allégeance implicites, car intériorisées par tous les membres de l'organisation, sont issues de l'ancienne pratique des tribus arabes, et possèdent un soubassement théologique et historique. Elles se fondent historiquement sur le serment de Hudaybiyya (Bay'at al-Ridwân), prêté par les musulmans au prophète Mahomet pour renouveler leur confiance et leur engagement à ses côtés. On trouve la mention de ce serment dans le Coran: "Ceux qui t'ont prêté serment, en vérité, ils le font avec Allah. La main d'Allah est sur leurs mains. Et ceux qui rompent le pacte le font contre eux. Et celui qui accomplit fidèlement son engagement, Allah lui apportera une récompense immense. » (sourate al-Fath, verset 10)

Le Coran met également en garde contre la rupture du serment d'allégeance: "Soyez fidèle au serment que vous avez prêté en prenant Allah à témoin; ne violez pas le serment après l'avoir contracté et désigné Allah comme garant pour vous. » (sourate $\mathrm{An}$-Nahl, verset 91)

Dans les traditions prophétiques (Hadith), le serment d'allégeance est même présenté comme obligatoire, en ce sens que tout croyant musulman doit le prêter à l'égard d'un chef musulman qui applique la charia car « celui qui meurt sans avoir prêté allégeance, meurt d'une mort digne de l'époque préislamique. » (Hadith rapporté par Muslim)

Plusieurs histoires authentifiées montrent que le serment d'allégeance possède plusieurs formes et qu'il peut être passé entre des personnes d'origines voire de sexes différents ou encore entre des groupes entiers qui désignent un chef pour les représenter ${ }^{7}$.

C'est ainsi que le groupe islamiste nigérian Boko Haram a prêté allégeance à AQMI en 2010. Comme preuve de cette allégeance " de groupe à groupe ", Boko Haram a émis le 2 octobre 2010 un communiqué appelant les Nigérians ${ }^{8}$ au djihad à travers l'organe médiatique d'AQMI, la société alAndalus ${ }^{9}$.

7. Voir Ibn Hisham, Sira, $1 / 141-143 ; 1 / 213$; 2/40-42 ; 2/47-56 ; Maqrizi, Imtâ' al-Asmâ', pp. 274-291; Ibn Taymiyya, Minhâj al-Sunna al-Nabawiyya, 5/330-331.

8. Rappelons que l'auteur de l'attentat manqué contre le vol Amsterdam-Détroit le jour de Noël 2009, Umar Faruq Abdulmutallab, était originaire du nord du Nigeria. Voir http://search.independent.co.uk/topic/umar-farouk-abdulmutallab-nigerian (juin 2011).

9. Voir http://www.bukisa.com/articles/366667_boko-haram-releases-eid-al-fitraddress-via-al-qaeda-in-north-africas-media-division-calling-on-muslims-to-wagejihad (juin 2011). 
En réalité, c'est le chef du groupe, le cheikh Mohammed Abou Bakr Ben Mohammed al-Shakwa, " émir du Groupe sunnite pour la prédication et le combat au Nigeria », alias Boko Haram, qui a prêté allégeance au chef d'AQMI, Abdelmalek Droukdal, engageant par là même l'ensemble de son groupe dans la mouvance d'Al-Qaïda.

Mais son allégeance répondait en réalité à la proposition d'aide faite par le chef d'AQMI quelques mois plus tôt. En effet, en février 2010, dans un message intitulé "Le génocide des musulmans au Nigeria: un nouvel épisode des croisades ", Droukdal proposait d'entraîner et d'armer les islamistes nigérians pour mener le djihad contre les chrétiens et les croisés au Nigeria et plus généralement en Afrique.

Cette proposition d'aide est aussi symbolique que pratique, et revêt à l'intérieur de la mouvance une importance cruciale parce qu'elle constitue le fondement même de son développement et de son expansion actuelle en Afrique subsaharienne. En généralisant le système d'allégeance, AQMI ouvre la voie à un " terrorisme par procuration », difficile à saisir au premier abord en raison de son caractère archaïque, mais qui s'avère d'une efficacité redoutable sur le terrain.

\section{LE TERRORISME PAR PROCURATION VU DE L'INTÉRIEUR}

Si le terrorisme par procuration est interprété de l'extérieur comme une compétition entre chefs, il en est tout autrement du point de vue interne à l'organisation. En réalité, il est le signe d'un renouvellement d'allégeance et d'une confirmation d'obéissance par l'acte.

Ainsi, dans son message de revendication des enlèvements d'Arlit, diffusé le 21 septembre 2010, le chef d'AQMI, Abdelmalek Droukdal, ne faisait que reprendre à son compte l'action de son vassal, l'émir du sud, Abdelhamid Abou Zeid, en vertu du serment d'allégeance que ce dernier lui avait prêté lors de sa nomination à la tête de la brigade Tarek Ben Ziyad ${ }^{10}$. Ce serment signifie que toutes les actions réalisées par Abou Zeid et sa brigade sont faites au nom du chef d'AQMI, Droukdal, et pour son compte, même si ce dernier ne participe pas personnellement ni à la planification ni à la conduite de ces actions et même s'il n'en donne pas l'ordre directement.

De même, dans son message du 27 octobre 2010 concernant les otages français, Ben Laden n'a fait que rappeler à AQMI, qui a commis ce kidnapping, et sans mentionner le nom de son chef Droukdal ni même celui de l'exécutant Abou Zeid, que tous deux lui devaient obéissance. Autrement dit, que les otages qu'ils détiennent appartiennent de droit au chef d'Al-Qaïda en personne, en vertu du serment d'allégeance qu'ils avaient prêté ${ }^{11}$.

10. Voir http://english.aljazeera.net/news/africa/2010/09/20109220826549357. html (juin 2011).

11. Voir http://articles.cnn.com/2010-10-27/world/france.bin.laden_1_rtl-radionew-tape-al-qaeda?_s=PM:WORLD (juin 2011). 
Trois semaines plus tard, le 18 novembre 2010 précisément, le chef d'AQMI répond à Ben Laden dans un enregistrement audio diffusé par la chaîne panarabe al-Jazeera. Dans cet enregistrement, Droukdal semble avoir bien compris le message du chef suprême d'Al-Qaïda. Outre le fait qu'il reprend mot pour mot les termes du " Message de Ben Laden au peuple français ${ }^{12}$ ", il renvoie le gouvernement français pour toute négociation concernant les otages au chef d'Al-Qaïda en personne: "Toute négociation pour la libération des otages devra être menée directement avec le lion de l'islam, notre chef à tous, Oussama Ben Laden ${ }^{13}$. »

La plupart des analystes sont alors surpris et ne comprennent pas le sens véritable de ce renvoi réciproque entre le chef d'Al-Qaïda et le chef d'AQMI. Les interprétations vont bon train: certains analystes commentent cela comme le signe d'une division interne, d'autres comme une opération de propagande visant à remettre Ben Laden sur le devant de la scène médiatique internationale. En réalité, ce ne sont là que des supputations sans preuve ni fondement. Du point de vue interne à l'organisation, il s'agit tout simplement d'un rappel du serment d'allégeance qui lie entre eux les différents chefs de l'organisation et qui leur impose certains droits et devoirs. Il existe ainsi une chaîne d'obédience qui va du chef d'Al-Qaïda jusqu'au plus petit caïd sur le terrain, sans qu'il n'y ait, à aucun moment, ni contact entre les différents acteurs ni ordre donné.

Il s'agit ainsi d'un système de commandement implicite qui tire sa force et son efficacité du système d'allégeance en vigueur au sein de l'organisation. Dans ce système, ce qui peut paraître parfois aléatoire ou même absurde vu de l'extérieur, possède une logique propre et une cohérence interne qu'il faut s'efforcer de déceler pour ne pas tomber dans l'interprétation ethnocentrique ou encore dans des biais cognitifs préjudiciables à la compréhension du phénomène ou à la prise de décision politique ou militaire.

Cela est d'autant plus important que ces logiques internes donnent lieu à des alliances conjoncturelles et à des formes inédites d'actions militaires qui sont souvent étrangères à la pensée occidentale en général et aux tactiques de guerre communément pratiquées dans les armées européennes et américaines. À cet égard, le cas libyen présente un exemple édifiant, en particulier depuis le lancement des opérations militaires à la suite de la résolution 1973 du Conseil de sécurité adoptée le 17 mars 2011.

\section{AQMI ET LA LiBYE}

Ayant été surpris, comme tout le monde, par la chute du régime de Ben Ali en Tunisie, le chef d'AQMI a bien essayé de se rattraper en diffusant en janvier

12. Voir http://english.aljazeera.net/news/africa/2010/11/201011194158585110. html (juin 2011).

13. Voir http://www.reuters.com/article/idUSTRE6AH60R20101118 (juin 2011). 
un " message à la jeunesse tunisienne » vibrant, qui est resté néanmoins sans effet. Mais l'organisation entend bien profiter de la détérioration de la situation sécuritaire dans le pays en envoyant des commandos d'éclaireurs et en tentant d'implanter des cellules terroristes. De fait, les infiltrations de groupes armés affiliés à AQMI se sont multipliées au cours des derniers mois. Ainsi, début mai 2011, les forces de sécurité ont arrêté dans le Sud du pays deux combattants de nationalité algérienne et libyenne. Le 18 mai 2011, ils ont abattu à Rouiha, dans le Centre-Ouest du pays, deux kamikazes qui portaient des ceintures d'explosifs et qui, bien qu'ils viennent d'Algérie, détenaient des passeports libyens. Un colonel de l'armée et deux soldats ont été tués au cours de cete opération. Des opérations de ratissage ont été organisées dans la région pour retrouver d'autres combattants infiltrés d'AQMI. En vain.

Car la Tunisie n'est pour l'heure qu'une zone de transit pour les combattants de l'organisation qui s'intéressent davantage aux événements en Libye et veulent à tout prix participer au combat à la fois contre le régime "impie » de Kadhafi et contre les forces " croisées ", si celles-ci venaient à s'engager sur le terrain. Début avril, le chef d'AQMI a émis deux messages dans lesquels il affirmait son soutien aux " frères libyens » à qui il promettait une aide pour chasser à la fois les Kadhafistes et les Occidentaux.

Dans cette optique, l'organisation a mis en œuvre une stratégie à trois volets: d'abord, encourager ses combattants d'origine libyenne à regagner leur pays pour combattre aux côtés de leurs " frères " (plusieurs groupes se seraient ainsi constitués depuis avril 2011); ensuite, récupérer des mains des trafiquants libyens le maximum d'armes (AK 47, RPG 7) et de matériels (pick-up et camions) dans les dépôts de Kadhafi (on parle même de missiles sol-air portables de type SAM-7); enfin, réactiver les anciens réseaux de l'organisation en Libye, en faisant appel aux membres du Groupe islamique combattant en Libye (GICL) et aux djihadistes revenus d'Irak.

Cet activisme d'AQMI à l'égard de la question libyenne inquiète au plus haut point les responsables occidentaux parce qu'il brouille les cartes en Libye et fait craindre une implantation durable de l'organisation dans le pays.

Début 2007, l'un des plus importants chefs libyens d'Al-Qaïda, Abou Layth al-Liby, avait dénoncé le revirement de Kadhafi et appelé au djihad contre lui : "Kadhafi c'est la tyrannie en Libye. Et voici qu'après tant d'années à s'opposer aux États-Unis, il découvre soudain que l'Amérique n'est pas un ennemi et transforme la Libye en une nouvelle base pour les croisés ». Dès lors, le même Abou al-Layth al-Liby, juste avant d'être tué lors d'un raid américain dans la province du Waziristan au Pakistan en 2008, s'est déclaré favorable à une alliance entre son groupe d'origine (GICL) et la nouvelle branche d'Al-Qaïda (AQMI).

Même si cette proposition avait suscité la controverse au sein du GICL, personne n'est surpris lorsque le 11 novembre 2007 l'Égyptien Ayman al- 
Zawahiri, alors numéro deux d'Al-Qaïda, annonce dans un enregistrement audio que le GICL a rejoint AQMI: "Ô Nation de l'islam au Maghreb, nation de la résistance et du djihad, voici que vos enfants s'unissent sous la bannière de l'islam et du djihad contre les Etats-Unis, la France et l'Espagne [...] pour nettoyer nos terres de leurs esclaves: Mouammar Kadhafi, Zine al-Abidine Ben Ali, Abdelaziz Bouteflika et Mohammed VI. »

Cette annonce vient confirmer l'intégration massive des djihadistes libyens au sein d'AQMI, en l'absence de perspective de lutte à l'intérieur de la Libye. Ceux-ci apportent à l'organisation leur expertise technique et notamment les acquis de leur expérience en Irak, terrain où ils ont représenté, pendant plusieurs années, le deuxième contingent de combattants étrangers après les Saoudiens ${ }^{14}$.

Les conséquences de cette intégration se font immédiatement sentir à travers la généralisation des attentats-suicide et la multiplication des attaques dans les pays du Sahel. Craignant des actions analogues en Libye, Kadhafi entreprend immédiatement des manœuvres tribales pour neutraliser le GICL. Pendant deux ans (2007-2009), il use de la carotte et du bâton: d'un côté, il mène une répression féroce contre les irréductibles ; de l'autre, il propose le dialogue et la réconciliation à ceux qui renoncent publiquement à la violence.

Cette stratégie donne des résultats probants: non seulement, il n'y a eu qu'un seul attentat en Libye au cours de cette période, alors qu'environ 500 étaient perpétrés dans le reste du Maghreb, mais en plus les cadres du GICL restés en Libye sont sommés de dissoudre le groupe. Ainsi, le 3 juillet 2009, durant le mois du ramadan, la direction du GICL publie une lettre ouverte intitulée « Études correctives sur la compréhension du djihad, sur sa recevabilité et sur le jugement du peuple ».

Au fond, il s'agit d'une révision des arguments juridiques islamiques visant à délégitimer le recours à la violence pour renverser les gouvernements arabes et musulmans.

Dans cette lettre, les principaux chefs du GICL reconnaissent leurs erreurs passées et critiquent les interprétations jugées contraires à l'islam, telles que les violences menées au nom du djihad ou les attaques contre les forces de sécurité. Mieux, les signataires y ont adjoint une lettre d'excuse adressée à Kadhafi en personne pour lui demander de les pardonner d'avoir tenté de l'assassiner au début des années 1990.

14. La Libye a été le premier pourvoyeur maghrébin de combattants à destination de l'Irak. À titre d'exemple, à l'est du pays, la seule ville de Darnah (80 000 habitants environ) a fourni, au cours de l'année 2005-2006, près de la moitié des combattants libyens engagés en Irak (52 sur 112). Dans le même temps, une ville comme Riyad, en Arabie Saoudite, pourtant gros pourvoyeur régional de djihadistes, n'a fourni que 51 combattants pour une population dépassant les 4 millions d'âmes. 
Cette lettre avait été précédée d'un communiqué publié en mars 2009 par le chef emprisonné du GICL, Abou Abdallah al-Sadiq, dans lequel il louait les efforts déployés par le fils de Kadhafi, Seïf al-Islam, pour faciliter les discussions avec le régime.

Même si avec le recul il paraît évident qu'aussi bien la lettre du GICL que les déclarations de son chef historique ont été obtenues sous la pression du régime, ce fut un coup dur pour AQMI qui vit se compromettre l'un de ses principaux alliés à l'est du Maghreb. Dans le journal libyen, Libya al-Mostaqbal, l' « appel à l'arrêt du djihad » est présenté comme l'acte de dissolution du GICL et la fin de l'islamisme radical en Libye.

À partir de cette date, le régime libyen engage un programme de " réhabilitation " par le biais de la Fondation Kadhafi dirigée par Seïf al-Islam, devenu entre-temps un interlocuteur bien connu des groupes djihadistes dans le monde. Ainsi, tout au long de l'année 2010 se succèdent les rapatriements de " repentis » et les libérations de militants « exilés » de la mouvance djihadiste. La prison d'Abou Salim à Tripoli, où des centaines de djihadistes avaient été exécutés en juin 1996, est quasiment vidée de ses détenus islamistes.

En mars 2010, sont libérés 214 prisonniers de différents groupes islamistes parmi lesquels $34 \mathrm{du}$ GICL dont ses trois chefs: Abdelhakim Belhaj, l'émir du groupe, Khaled Charif, le chef militaire, et Sami Saadi, l'idéologue attitré.

Le $1^{\mathrm{er}}$ septembre 2010, à la veille de la célébration du $41^{\mathrm{e}}$ anniversaire de la révolution libyenne qui a porté le colonel Kadhafi au pouvoir, son fils Seïf alIslam annonce une série de libérations d'islamistes dont un ancien chauffeur de Ben Laden, Nasser Tailamoun, et un ex-détenu de Guantanamo, Abou Sofiane Ben Guemou, livré par les Américains en 2007. De source officielle, le reste des islamistes libérés, soit plus de 200 détenus, sont des membres du GICL ayant collaboré avec Al-Qaïda en Irak ou au Maghreb.

À six mois du soulèvement de février 2011, ces libérations ont été précédées par des débats menés dans les prisons, sous la supervision du régime, entre jeunes militants islamistes désireux de participer - ou ayant participé - à ce qu'ils considèrent comme le djihad obligatoire, soit en Irak, soit en Afghanistan, ou encore en Somalie. Il en ressort que la jeune génération de "néo-djihadistes » est différente sur bien des points de la vieille garde du GICL, et soutient ouvertement Al-Qaïda et sa stratégie contre les Occidentaux. C'est là un mauvais signe pour l'avenir des relations avec la « nouvelle Libye » en construction.

Enfin, le 17 février 2011, alors même que l'Est du pays préparait une « journée de la colère », le régime croit amadouer les tribus de la Cyrénaïque en annonçant la libération de 110 prisonniers du GICL issus de la région, ce qui porte à 946 le nombre total d'islamistes radicaux libérés depuis le début du programme « dialogue et réconciliation » initié par le régime. Mais 
le plus inquiétant dans ce programme est que la totalité du processus de " réhabilitation » est mue par des rivalités internes au clan Kadhafi, étant donné la question ouverte de la succession du "guide de la révolution ». Ainsi, Seiff al-Islam a instrumentalisé ce programme de « réhabilitation » à des fins politiques, pour apparaître à l'intérieur comme l'homme fort de son clan et à l'extérieur comme l'artisan de la réconciliation avec la Cyrénaïque. Cela signifie que la question de l'avenir de la mouvance djihadiste libyenne demeure entière: que faut-il en faire? Quel avenir pour ses membres? D'autant plus que les expériences saoudiennes et yéménites de "réhabilitation » ont démontré leurs limites: même les ex-djihadistes qui ont accepté de renoncer à la violence continuent, dans les faits, de propager des idées radicales.

Le 23 février 2011, par la voix de son vice-ministre des Affaires étrangères, le régime libyen annonçait au monde entier qu'Al-Qaïda avait établi un émirat islamiste à Darnah et qu'elle envisageait la mise en place d'un État « à la talibane ». Au même moment, dans un message audio, le chef d'AQMI, Abdelmalek Droukdal, assurait aux manifestants libyens son soutien total et leur promettait de tout faire pour les aider à se débarrasser du colonel Kadhafi: " Nous ferons tout notre possible pour vous aider, avec le pouvoir d'Allah, car votre combat est le combat de tout musulman qui aime Allah et son messager. »

Le 6 mars 2011, le colonel Kadhafi aux abois déclare à un journal français: " Il y aura un djihad islamique en face de vous, en Méditerranée. [...] Les gens de Ben Laden viendront imposer des rançons sur terre et sur mer. On reviendra au temps de Barberousse, des pirates, des Ottomans qui imposaient des rançons sur les bateaux. Ce sera vraiment une crise mondiale et une catastrophe pour tout le monde. »

Force est de constater que Kadhafi a toujours su jouer de la menace terroriste: il a utilisé les islamistes quand il en avait besoin pour asseoir sa politique intérieure et décimé leurs rangs et quand cela servait sa politique extérieure, oscillant ainsi entre répression féroce et amnistie générale.

Depuis avril 2011, l'un des chefs du GICL, Abdelhakim al-Hasidi, ancien d'Afghanistan, est désormais membre du Conseil national transitoire (CNT) de Dernah et il est également responsable de la sécurité de l'Est de la Cyrénaïque. Il opère sous les ordres du général Abdelfattah Younis, ancien ministre de l'Intérieur de Kadhafi, et disposerait d'un millier d'hommes sous son commandement, pour la plupart des islamistes et des anciens djihadistes. Ils sont considérés comme un pilier de l'opposition armée à l'Est du pays et plusieurs sont morts dans les combats contre les forces de Kadhafi notamment à Brega et à Misrata.

Dans une interview accordée le 16 avril 2011 au journal saoudien alHayyat publié à Londres, le porte-parole d'AQMI, Saleh Abou Mohammad, a confirmé que son organisation disposait de contacts et de combattants 
sur le terrain, et qu'elle visait à terme l'instauration d'un émirat islamiste à l'Est du pays ${ }^{15}$.

Certes, on pourrait imaginer AQMI s'implanter durablement en Libye à la faveur de l'insurrection armée ou encore voir le GICL renaître sous un autre nom comme force politique ayant la légitimité de plusieurs années d'opposition à Kadhafi. Mais un régime islamiste radical n'est possible en Libye que si les chefs des principales tribus se rallient à la cause djihadiste, ce qui paraît peu probable aujourd'hui.

Bref, l'évocation du péril islamiste en Libye n'est pas totalement farfelue, mais la situation ethnique du pays est bien trop complexe pour que les islamistes puissent s'emparer aisément du pouvoir. Même si le vide étatique peut bénéficier à AQMI et à ses sympathisants libyens, ce sont les tribus qui contrôlent en définitive les territoires et, à ce titre, elles ne laisseront aucune force exogène en prendre le contrôle. En un mot, les islamistes libyens sont probablement les mieux armés aujourd'hui pour peser sur l'avenir politique de la Libye mais ils devront composer, comme tout le monde avant eux, avec la dimension tribale et clanique du pays.

\section{AQMI ET LE « PRINTEMPS ARABE »}

Depuis sa création, AQMI s'est fixée comme objectif la chute des régimes actuels du Maghreb, en particulier le régime algérien, et a pour cela adopté une stratégie prioritairement "indirecte » en attaquant les soutiens de ces régimes (les Occidentaux, la France en tête). Le chef d'AQMI est en effet convaincu que les régimes en question tiennent uniquement grâce au soutien économique, financier et militaire de l'Occident (Europe et États-Unis).

Les manifestations récentes dans ces pays ne visent en définitive pas autre chose que la chute des régimes en place, mais il est vrai que pour l'heure, les manifestants ne se réclament pas de l'islamisme radical et ne prennent pas pour cibles les Occidentaux parce que l'enjeu est local et conjoncturel: faire tomber le plus rapidement possible un dictateur ou un clan qui accapare le pouvoir parfois depuis des décennies. Mais sur le long terme il n'est pas certain que cette hostilité à l'égard de l'Occident ne refasse pas surface de façon exacerbée, étant donné la persistance des mêmes rapports de force aux niveaux national et régional.

Ainsi, il serait plus juste d'affirmer à ce stade des événements que les soulèvements populaires constituent une décrédibilisation de la stratégie et des modes d'action d'Al-Qaïda mais certainement pas une négation de l'objectif ni des finalités politiques de la mouvance dans son ensemble. C'est ce qui explique d'ailleurs le soutien direct du nouveau chef de l'organisation, Ayman al-Zawahiri, aux soulèvements en cours dans son dernier message de

15. Voir l'intégralité de l'interview en arabe ici : http://www.algeriachannel. net/?p=5531 (juin 2011). 
juin 2011. Pour lui, « tous les frères rebelles égyptiens, libyens, tunisiens ou syriens " poursuivent les mêmes objectifs qu'Al-Qaïda, même s'ils n'utilisent pas les mêmes mots ni les mêmes moyens.

Il convient de noter néanmoins que depuis le déclenchement des révolutions arabes et surtout depuis les succès tunisien et égyptien, il existe indéniablement un débat interne à l'organisation quant à la justification du djihad et sur sa place par rapport à la protestation populaire. On le perçoit clairement dans les interrogations des militants et des sympathisants sur les forums Internet, sur les blogs et sur les messages postés sur Facebook ou sur Twitter en soutien aux manifestations dans les différents pays. Le point central du débat porte sur l'utilité du djihad dans la phase actuelle de l'histoire de la Oumma, puisque les régimes vacillent sous la pression de l'action pacifique de la masse et non pas sous l'effet de l'action violente d'un groupe.

Certains se posent la question de savoir s'il n'est pas plus opportun de rejoindre les manifestants dans les rues pour défiler pacifiquement plutôt que d'attendre l'heure de la confrontation armée dans le maquis ou dans le désert. Face à ces questionnements militants qui s'apparentent malgré tout à un doute existentiel, les différents chefs d'Al-Qaïda, dont Zawahiri et Droukdal, ont successivement apporté une réponse articulée que l'on peut résumer ainsi :

Primo, à ce stade des événements, le djihad est utile pour soutenir l'action des manifestants en portant des coups durs aux régimes en place par des actions militaires ciblées (ce qui explique par exemple l'intensification des actions d'AQMI en Algérie). Deuzio, le djihad sera utile par la suite pour défendre les acquis de la révolution en attaquant toute personne qui essaierait de la récupérer à son compte en excluant les islamistes (ce qui explique par exemple les tentatives d'implantation d'AQMI en Tunisie). Tertio, le djihad sera utile sur le long terme pour sécuriser la révolution et permettre la réalisation des objectifs politiques de l'organisation en instaurant la charia et en chassant les Occidentaux des pays musulmans. En effet, les différents " émirs » régionaux d'Al-Qaïda, à commencer par Droukdal au Maghreb, parient sur le maintien sous une nouvelle forme de l'ancien régime, et mettent en garde les populations contre l'Occident qui, selon eux, placera au final d'autres " agents à sa solde » à la tête des pays du Maghreb et du Machrek. C'est pourquoi ils estiment que le djihad sera l'ultime rempart contre l'échec de ces révolutions dont ils se présentent désormais à la fois comme les inspirateurs et les défenseurs.

\section{Conclusion}

On le voit, six mois à peine après la révolution tunisienne, AQMI tente de se remettre en selle en justifiant sa présence sur le court, le moyen et le long terme. Pour l'instant, cela semble convaincre les militants mais il est évident que cela dépendra de l'issue des protestations en cours dans les autres pays 
du Maghreb. En cas d'échec il ne fait aucun doute qu'AQMI en sortira renforcée en récupérant tous les déçus du mouvement révolutionnaire qui iraient grossir ses rangs, convaincus qu'il n'existe pas d'autre solution que la lutte armée après tant d'efforts et de sang versé en vain.

En revanche, si ces révolutions réussissent, c'est-à-dire si elles aboutissent non seulement à un changement de régimes mais aussi à un changement des pratiques et des rapports de force, AQMI sera en mauvaise posture pour justifier la poursuite de ses actions violentes et sa promotion du terrorisme, en particulier au sein des pays musulmans. Même en cas de succès partiel, par exemple en Tunisie, preuve sera faite de la possibilité d'un changement effectif dans un pays arabe sans recours à la violence aveugle et sans exacerbation de la haine anti-occidentale.

Il est donc encore trop tôt pour juger de l'évolution à long terme de l'organisation, mais il est clair que cette évolution sera tributaire du positionnement diplomatique international par rapport au monde arabomusulman, car Al-Qaïda a fait de la lutte contre les États-Unis et ses alliés le fondement de sa légitimité et la clé de voûte de sa stratégie de combat. On l'a vu en 2009, le discours réconciliateur d'Obama a déstabilisé pendant un moment l'organisation et a laissé les militants perplexes quant à la position qu'il convient d'adopter face aux États-Unis. Sur le terrain, la poursuite des guerres en Irak et en Afghanistan ainsi que la persistance de certains discours va-t'en guerre n'ont pas permis de transformer cette perplexité interne en une victoire idéologique des démocraties. Elles ont plutôt offert à l'organisation la possibilité d'exploiter les mêmes arguments de justification de son terrorisme: ingérence de l'Occident, pillage des ressources naturelles, victimes civiles musulmanes, question palestinienne. C'est dans ces pseudo-arguments qui motivent le soutien et la poursuite des objectifs de l'organisation qu'il faut chercher la solution à long terme: d'une part par une action concertée de contre-propagande et d'autre part par l'affichage de positions politiques et diplomatiques justes et transparentes. En tout état de cause, si l'on veut la fin d'Al-Qaïda, il faut définitivement sortir de la logique de confrontation, instaurée après le 11 septembre 2001, entre les néoconservateurs américains et les néofondamentalistes musulmans.

Au Maghreb, numériquement comme militairement, AQMI ne représente plus grand-chose aujourd'hui et ne constitue pas une menace mortelle pour l'Europe; elle ne possède pas non plus l'ancrage territorial et le soutien populaire qu'elle espérait dans les pays du Sahel, c'est pourquoi il faut s'intéresser à d'autres rapports de force et initier, à la faveur des révolutions arabes, de nouveaux partenariats stratégiques. 
